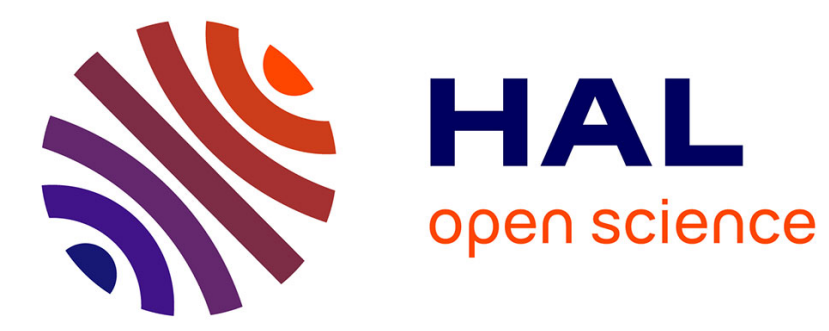

\title{
The Second-order Parametric Consensus Protocol
}

Fabio Morbidi

\section{To cite this version:}

Fabio Morbidi. The Second-order Parametric Consensus Protocol. ECC 2014 - 13th European Control Conference, Jun 2014, Strasbourg, France. pp.202-207. hal-00979666

\section{HAL Id: hal-00979666 https://hal.science/hal-00979666}

Submitted on 16 Apr 2014

HAL is a multi-disciplinary open access archive for the deposit and dissemination of scientific research documents, whether they are published or not. The documents may come from teaching and research institutions in France or abroad, or from public or private research centers.
L'archive ouverte pluridisciplinaire HAL, est destinée au dépôt et à la diffusion de documents scientifiques de niveau recherche, publiés ou non, émanant des établissements d'enseignement et de recherche français ou étrangers, des laboratoires publics ou privés. 


\title{
The Second-order Parametric Consensus Protocol
}

\author{
Fabio Morbidi
}

\begin{abstract}
In this paper we extend the parametric consensus protocol recently introduced by the author, to more realistic agents modeled as double integrators and interacting over an undirected communication network. The stability properties of the new protocol in terms of the real parameter " $s$ " are studied for some relevant graph topologies, and the connection with the notion of bipartite consensus is highlighted. The theory is illustrated with the help of two worked examples, dealing with the coordination of a team of quadrotor UAVs and with cooperative temperature measurement in an indoor environment.
\end{abstract}

\section{INTRODUCTION}

\section{A. Motivation and related work}

In recent years, a large body of research has started to explore the system-theoretic properties of the consensus protocol [1], such as its robustness against communication delays, noisy measurements and switching topologies [2], [3], and - by introducing suitable input/output signals - its controllability and observability properties with applications to formation control and network reconstruction/security (see, e.g., [4]-[8] and the references therein). In particular, considerable effort has been invested in relating these properties to the topology of the underlying communication network. However, the set of behaviors unfolding from the interaction of system- and graph-theoretic properties has not been yet fully investigated, and a general theory has remained so far elusive.

Following this vibrant line of research and going a step further toward the development of such a theory, recent works of the author [9], [10], have opened the doors to the study of the so-called parametric consensus protocols, i.e. continuoustime consensus protocols whose stability properties depend on the value of a real parameter " $s$ " which scales the information coming from the neighbors of each node in the network. In this class of protocols, the inter-node couplings are not diffusive (cf. [5]), and parameter " $s$ " can be regarded as an "external input" that can be utilized to trigger different behaviors in the multi-agent system, thus giving the designer enhanced freedom. As shown in [9], this is particularly useful when the motion of a team of autonomous vehicles needs to be coordinated by a (human) supervisor, or when a wireless sensor network needs to adapt its operation over time in response to changes in the environment.

\section{B. Original contributions and organization}

In this work, we extend the results in [10] to agents modeled as double integrators. The double integrator can be used, indeed, to model numerous real systems: in fact, the simplified dynamics of a quadrotor UAV (Unmanned

The author is with the "Networked Controlled System" (NeCS) team, Inria Grenoble Rhône-Alpes, 655 Avenue de l'Europe, 38334 Montbonnot Saint Martin, France. Email: fabio.morbidi@inria.fr
Aerial Vehicle), or the dynamics of a unicycle robot (after being dynamically feedback linearized) comply with this model. The stability properties of the second-order parametric consensus protocol are studied for some special families of undirected graphs, in terms of parameter " $s$ ". The case of bipartite graphs and its connection with the properties of the signless Laplacian matrix [11], [12], is also discussed. Although the analysis for general graph topologies is challenging, the matrix pencil formalism introduced in [10] appears still useful for shedding some light into the stability properties of the new protocol.

Research on second-order consensus algorithms has been active since the publication of the seminal contributions of Ren et al. [13] and Yu et al. [14]. In this paper, some of the results in [13] are revisited in a more general setting. Two worked examples, dealing with the coordination of a team of quadrotor UAVs and with cooperative temperature measurement in a bounded environment, are provided to illustrate the proposed theory and show its applicative potential.

The rest of this paper is organized as follows. Sect. II presents some preliminaries on algebraic graph theory and introduces the notation. Sect. III is devoted to the problem formulation, and the main theoretical results of the paper are provided in Sect. IV. The worked examples are discussed in Sect. V. Finally, Sect. VI summarizes the main contributions of the paper and outlines possible future research directions.

\section{PRELIMINARIES}

In this section we briefly review some basic notions of algebraic graph theory and introduce the notation used through the paper. Let $\mathcal{G}=(V, E)$ be a graph where $V=\{1, \ldots, n\}$ is the set of nodes, and $E$ is the set of edges. All $\mathcal{G}$ are assumed to be "schlicht" graphs, i.e., finite undirected graphs without self-loops and multiple edges.

Definition 1 (Bipartite graph): A graph $\mathcal{G}$ is called bipartite if its set of nodes $V$ can be divided into two disjoint sets $V_{1}$ and $V_{2}$, such that every edge connects a node in $V_{1}$ to one in $V_{2}$. If $\mathcal{G}$ is connected, its bipartition $\left\{V_{1}, V_{2}\right\}$ is unique.

Definition 2 ( $\kappa$-regular graph): A graph $\mathcal{G}$ is called $\kappa$-regular, when every node has precisely $\kappa \leq n-1$ neighbors.

Definition 3 (Adjacency matrix A): The adjacency matrix $\mathbf{A}=\left[a_{i j}\right]$ of graph $\mathcal{G}$ is an $n \times n$ matrix defined as $a_{i j}=1$ if $\{i, j\} \in E$ and $a_{i j}=0$ otherwise.

Definition 4 (Laplacian matrix L): The Laplacian matrix of graph $\mathcal{G}$ is an $n \times n$ symmetric positive semidefinite matrix defined as $\mathbf{L}=\mathbf{D}-\mathbf{A}$ where $\mathbf{D}=\operatorname{diag}(\mathbf{A} \mathbb{1})$ is the degree matrix and $\mathbb{1}$ is a column vector of $n$ ones.

Definition 5 (Signless Laplacian matrix Q [11]):

The signless Laplacian matrix of graph $\mathcal{G}$ is an $n \times n$ matrix defined as $\mathbf{Q}=\mathbf{D}+\mathbf{A}$. 
As $\mathbf{L}$, the signless Laplacian $\mathbf{Q}$ is a symmetric positive semidefinite matrix, but it is not necessarily singular.

Property 1 (Spectral properties of Q [11], [15]):

1) Let $\mathcal{G}$ be a $\kappa$-regular graph. Then $p_{\mathbf{L}}(\lambda)=$ $(-1)^{n} p_{\mathbf{Q}}(2 \kappa-\lambda)$ where $p_{\mathbf{L}}(\lambda)$ denotes the characteristic polynomial of the Laplacian $\mathbf{L}$.

If $\mathcal{G}$ is a bipartite graph, then $p_{\mathbf{L}}(\lambda)=p_{\mathbf{Q}}(\lambda)$.

2) The least eigenvalue of $Q$ of a connected graph is equal to 0 if and only if the graph is bipartite. In this case, 0 is a simple eigenvalue and the corresponding eigenvector is $\mathbf{v}=\left[v_{k}\right]$ where $v_{i}=1, i \in V_{1}$ and $v_{j}=-1, j \in V_{2}$, being $\left\{V_{1}, V_{2}\right\}$ the bipartition of $\mathcal{G}$.

3) In any graph, the multiplicity of the eigenvalue 0 of $\mathrm{Q}$ is equal to the number of bipartite connected components of $\mathcal{G}$.

In a sense, the least eigenvalue of $\mathrm{Q}$ can be interpreted as a measure of how close $\mathcal{G}$ is to being a bipartite graph. A similar idea has been utilized for the second-smallest eigenvalue of $\mathbf{L}$, which is known as the algebraic connectivity of $\mathcal{G}$ [16]. For this reason, the authors of [17] call the least eigenvalue of $\mathbf{Q}$ the algebraic bipartiteness of $\mathcal{G}$.

Notation: In the reminder of the paper, $\mathrm{k} \triangleq$ $\left[-1,1,-1,1, \ldots,(-1)^{n-1},(-1)^{n}\right]^{T} \in \mathbb{R}^{n}, \mathbf{0}_{n}$ will denote the $n \times n$ zeros matrix, $\mathbf{I}_{n}$ the $n \times n$ identity matrix, $\mathrm{J}_{m \times n}$ the $m \times n$ ones matrix, $\otimes$ the Kronecker product, $\lfloor\cdot\rfloor$ the floor function which maps a real number to the largest previous integer, $|\mathcal{B}|$ the cardinality of the set $\mathcal{B}$, and $\operatorname{deg}[p(s)]$ the degree of the polynomial with real coefficients $p(s)$.

\section{PROBLEM FORMULATION}

For agents modeled by

$$
\dot{x}_{i}=u_{i}, \quad i \in\{1, \ldots, n\},
$$

where $x_{i} \in \mathbb{R}$ is the state of agent $i$ and $u_{i} \in \mathbb{R}$ its input, a first-order consensus protocol was proposed in [2], as

$$
u_{i}=\sum_{j \in \mathcal{N}(i)}\left(x_{j}-x_{i}\right), \quad i \in\{1, \ldots, n\},
$$

where $\mathcal{N}(i)$ denotes the set of nodes adjacent to node $i$ in the undirected communication graph $\mathcal{G}$. By applying control (2), equation (1) can be rewritten in matrix form as $\dot{\mathbf{x}}=-\mathbf{L ~} \mathbf{x}$ where $\mathbf{x} \triangleq\left[x_{1}, \ldots, x_{n}\right]^{T} \in \mathbb{R}^{n}$. If the graph $\mathcal{G}$ is connected, it is well known that $\mathbf{x}$ asymptotically converges to $\frac{1}{n} \sum_{i=1}^{n} x_{i}(0)=\frac{1}{n} \mathbf{x}_{0}^{T} \mathbb{1}$, where $\mathbf{x}_{0} \triangleq\left[x_{1}(0), \ldots, x_{n}(0)\right]^{T}$ is the vector of initial states. In this paper, we consider a double-integrator model for the agents,

$$
\dot{x}_{i}=\nu_{i}, \quad \dot{\nu}_{i}=u_{i}, \quad i \in\{1, \ldots, n\},
$$

where $\left[x_{i}, \nu_{i}\right]^{T} \in \mathbb{R}^{2}$ is the state vector of agent $i$ and $u_{i} \in \mathbb{R}$ its input, and inspired by [10], [13] we propose the following second-order parametric consensus protocol:

$$
u_{i}=\sum_{j \in \mathcal{N}(i)}\left[\left(s x_{j}-x_{i}\right)+\gamma\left(s \nu_{j}-\nu_{i}\right)\right], i \in\{1, \ldots, n\},
$$

where $\gamma$ is a positive constant and $s$ is a real parameter. By applying control (4), system (3) can be rewritten in compact form as:

$$
\left[\begin{array}{c}
\dot{\mathbf{x}} \\
\dot{\nu}
\end{array}\right]=-\boldsymbol{\Gamma}(s)\left[\begin{array}{l}
\mathbf{x} \\
\nu
\end{array}\right]
$$

where $\boldsymbol{\nu} \triangleq\left[\nu_{1}, \ldots, \nu_{n}\right]^{T} \in \mathbb{R}^{n}$,

$$
\boldsymbol{\Gamma}(s)=\left[\begin{array}{cc}
\mathbf{0}_{n} & -\mathbf{I}_{n} \\
\mathbf{L}_{p}(s) & \gamma \mathbf{L}_{p}(s)
\end{array}\right],
$$

and $\mathbf{L}_{p}(s)=\mathbf{D}-s \mathbf{A}$ is called the parametric Laplacian [10]. Note that $\mathbf{L}_{p}(s) \in \mathbb{R}^{n \times n}$ is a symmetric matrix (but not positive semidefinite as $\mathbf{L}$, in general), and that $\mathbf{L}_{p}(1)=\mathbf{L}, \mathbf{L}_{p}(-1)=\mathbf{Q}$. Assuming that the graph $\mathcal{G}$ is connected, our objective is to study the stability properties of system (5) in terms of the real parameter $s$. We will say that consensus is reached asymptotically if,

$\lim _{t \rightarrow \infty}\left|x_{j}(t)-x_{i}(t)\right|=0, \lim _{t \rightarrow \infty}\left|\nu_{j}(t)-\nu_{i}(t)\right|=0, \forall j \neq i$,

for any initial conditions $\mathbf{x}_{0}$ and $\nu_{0} \triangleq\left[\nu_{1}(0), \ldots, \nu_{n}(0)\right]^{T}$.

Note that in the special case of $s=1$, we have the following result (cf. Lemma 4.1 and 4.2 in [13]).

Lemma 1: If $s=1$, system (5) achieves consensus asymptotically for any $\gamma>0$. In particular, for large $t$, we have that,

$$
\mathbf{x}(t) \rightarrow \frac{1}{n} \mathbb{1}^{T}\left(\mathbf{x}_{0}+\boldsymbol{\nu}_{0} t\right) \quad \text { and } \quad \boldsymbol{\nu}(t) \rightarrow \frac{1}{n} \mathbb{1}^{T} \boldsymbol{\nu}_{0}
$$

In fact, the eigenvalues of $\mathbf{L}_{p}(s)$ and $\boldsymbol{\Gamma}(s)$ are closely related, as shown by the next lemma (see [13, p. 1007]).

Lemma 2: Let $\varrho_{i}(s)$ be the $i$-th eigenvalue of $-\mathbf{L}_{p}(s)$. Then, the eigenvalues of $-\boldsymbol{\Gamma}(s)$ are:

$$
\lambda_{i \pm}(s)=\frac{\gamma \varrho_{i}(s) \pm \sqrt{\gamma^{2} \varrho_{i}^{2}(s)+4 \varrho_{i}(s)}}{2}, i \in\{1, \ldots, n\},
$$

where $\lambda_{i+}(s)$ and $\lambda_{i-}(s)$ are the eigenvalues of $-\boldsymbol{\Gamma}(s)$ associated to $\varrho_{i}(s)$.

Another special case worth mentioning is the following (cf. [12, Th. 5] and recall item 2 of Property 1).

Proposition 1: Consider the system $\dot{\mathbf{x}}=-\mathbf{Q} \mathbf{x}$. If the graph $\mathcal{G}$ is connected and bipartite with bipartition $\left\{V_{1}, V_{2}\right\}$, we have that,

$$
\lim _{\substack{t \rightarrow \infty \\ i \in V_{1}}} x_{i}(t)=-\lim _{\substack{t \rightarrow \infty \\ j \in V_{2}}} x_{j}(t)=\frac{1}{n}\left(\sum_{i \in V_{1}} x_{i}(0)-\sum_{j \in V_{2}} x_{j}(0)\right),
$$

and the quantity $\sum_{i \in V_{1}} x_{i}(t)-\sum_{j \in V_{2}} x_{j}(t)$ is time invariant.

This behavior is referred to as bipartite consensus in [18] and cluster anticonsensus in [12]: in fact the states of the nodes in $V_{1}$ and $V_{2}$ asymptotically assume the same absolute value but have opposite signs.

In the next section, we will focus on some special families of graphs for which the eigenvalues and eigenvectors of $\boldsymbol{\Gamma}(s)$ can be computed in closed form and thus the stability properties of system (5) can be easily established.

\section{STABILITY CONDITIONS FOR SOME SPECIAL FAMILIES OF GRAPHS}

This section presents a sequence of seven propositions, providing stability conditions for system (5) in the case of path, cycle, $m$-cube (or hypercube), Petersen, complete, complete bipartite, and Hamming graphs (the reader is referred to [16], [19] for a precise definition of these graphs). 
Proposition 2 (Path graph $P_{n}$ ): For the path graph $P_{n}$ with $n \geq 2$ nodes (we number the nodes from 1 to $n$ in the natural order from left to right), we have that:

- For $|s|<1$, system (5) is asymptotically stable.

- For $|s|>1$, system (5) is unstable.

- For $s=-1$ and for large $t$ we have that:

$$
\mathbf{x}(t) \rightarrow \frac{1}{n} \mathbb{k}_{k} \mathbb{k}^{T}\left(\mathbf{x}_{0}+\boldsymbol{\nu}_{0} t\right) \text { and } \boldsymbol{\nu}(t) \rightarrow \frac{1}{n} \mathbb{k}_{k} \mathbb{k}^{T} \nu_{0} .
$$

Proof: Using the Sturm sequence property, it has been shown in [10] that the eigenvalues $\varrho_{i}(s)$ of $-\mathbf{L}_{p}(s)$ are strictly negative for $|s|<1$. But from Lemma 2, being $\left|\gamma \varrho_{i}(s)\right|>\left|\sqrt{\gamma^{2} \varrho_{i}^{2}(s)+4 \varrho_{i}(s)}\right|$ for $\varrho_{i}(s)<0, i \in$ $\{1, \ldots, n\}$, it follows that the eigenvalues $\lambda_{i \pm}(s)$ of $-\boldsymbol{\Gamma}(s)$ have negative real parts for $|s|<1$, thus proving the first two items of the statement. The third item follows from Prop. 1.

Proposition 3 (Cycle graph $C_{n}$ ): For the cycle graph $C_{n}$ with $n>2$ nodes (we number the nodes from 1 to $n$ in the natural order, moving counterclockwise), we have that:

- If $n$ is even:

- For $|s|<1$, system (5) is asymptotically stable.

- For $|s|>1$, system (5) is unstable.

- For $s=-1$ and for large $t$, the state vector of system (5) obeys (6).

- If $n$ is odd, let $\mu \triangleq 1 / \cos \left(\frac{n+1}{n} \pi\right)$. Then:

- For $s \in(\mu, 1)$, system (5) is asymptotically stable.

- For $s<\mu$ or $s>1$, system (5) is unstable.

- For $s=\mu$, the components of the state subvector $\nu$ of system (5) asymptotically converge, in general, to $n$ different values.

Proof: Since the eigenvalues of $-\mathbf{L}_{p}(s)$ are (cf. [10]), $2 \cos \left(\frac{2 \pi(i-1)}{n}\right) s-2, i \in\{1, \ldots, n\}$, invoking Lemma 2 , we have that the eigenvalues of $-\boldsymbol{\Gamma}(s)$ are, for $i \in$ $\{1, \ldots, n\}$ :

$\gamma\left[\cos \left(\frac{2 \pi(i-1)}{n}\right) s-1\right]$

$\pm \sqrt{\gamma^{2} \cos ^{2}\left(\frac{2 \pi(i-1)}{n}\right) s^{2}+2\left(1-\gamma^{2}\right) \cos \left(\frac{2 \pi(i-1)}{n}\right) s+\gamma^{2}-2}$

From a systematic study of (7) for variable $s$, the first two bullets of each item in the statement are immediately proved. Finally, if $n$ is even and $s=-1$ we can utilize Prop. 1, and if $n$ is odd and $s=\mu$, for large $t$ we have that:

$$
\begin{gathered}
\exp (-\boldsymbol{\Gamma}(\mu) t) \rightarrow\left[\begin{array}{cc}
\mathbf{M} & \mathbf{M} t \\
\mathbf{0}_{n} & \mathbf{M}
\end{array}\right], \\
\mathbf{M}=\frac{2}{n} \operatorname{circ}\left[\cos \left(\frac{2 \pi\lfloor n / 2\rfloor \cdot 0}{n}\right), \cos \left(\frac{2 \pi\lfloor n / 2\rfloor \cdot 1}{n}\right),\right. \\
\left.\cos \left(\frac{2 \pi\lfloor n / 2\rfloor \cdot 2}{n}\right), \ldots, \cos \left(\frac{2 \pi\lfloor n / 2\rfloor \cdot(n-1)}{n}\right)\right],
\end{gathered}
$$

and $\operatorname{circ}[\cdot]$ denotes a circulant matrix [20], i.e. each subsequent row of the matrix is simply the row above shifted one element to the right (and wrapped around, i.e. modulo $n$ ).

Proposition 4 (m-cube $\left.Q_{m}\right)$ : For the $m$-cube (or hypercube) graph $Q_{m}$ with $n=2^{m}>4$ nodes, we have that:

- For $|s|<1$, system (5) is asymptotically stable.

- For $|s|>1$, system (5) is unstable.

- For $s=-1$ and for large $t$, the state vector of system (5) obeys (6).
Proof: Since the eigenvalues of $-\mathbf{L}_{p}(s)$ are in this case, $-m+s(m-2 \ell)$ with multiplicity $\left(\begin{array}{c}m \\ \ell\end{array}\right)$, $\ell \in\{0, \ldots, m\}$, from Lemma 2, we have that the eigenvalues of $-\boldsymbol{\Gamma}(s)$ are $[\gamma(-m+s(m-2 \ell)) \pm$ $\left.\sqrt{\gamma^{2}(-m+s(m-2 \ell))^{2}+4(-m+s(m-2 \ell))}\right] / 2$ with multiplicity $\left(\begin{array}{c}m \\ \ell\end{array}\right)$. It is easy to verify that these eigenvalues are all negative only for $|s|<1$, which proves the first two items of the statement. For the third item, we can rely on Prop. 1, after a suitable labeling of the nodes of $Q_{m}$.

Proposition 5 (Petersen graph $J(5,2,0)$ ):

For the Petersen graph, we have that:

- For $s \in(-3 / 2,1)$, system (5) is asymptotically stable.

- For $s>1$ or $s<-3 / 2$, system (5) is unstable.

- For $s=-3 / 2$, the components of the state subvector $\nu$ of system (5) asymptotically converge, in general, to ten different values.

Proof: Since the eigenvalues of $-\mathbf{L}_{p}(s)$ are in this case, $3 s-3$ with multiplicity $1, s-3$ with multiplicity 5 and $-2 s-3$ with multiplicity 4 , from Lemma 2 we have that the eigenvalues of $-\boldsymbol{\Gamma}(s)$ are $[\gamma(3 s-3) \pm$ $\left.\sqrt{\gamma^{2}(3 s-3)^{2}+4(3 s-3)}\right] / 2$ with multiplicity $1,[\gamma(s-$ $\left.3) \pm \sqrt{\gamma^{2}(s-3)^{2}+4(s-3)}\right] / 2$ with multiplicity 5 , and $\left[-\gamma(2 s+3) \pm \sqrt{\gamma^{2}(2 s+3)^{2}-4(2 s+3)}\right] / 2$ with multiplicity 4 , from which the first two items of the statement follow. For the third item, by numbering the nodes from 1 to 5 and from 6 to 10 in the natural order moving counterclockwise along the external and internal "ring" of the graph, (cf. [19, Fig. 1.8]), we have that for large $t$,

$\exp (-\boldsymbol{\Gamma}(-3 / 2) t) \rightarrow\left[\begin{array}{cc}\frac{1}{3}\left(\mathbf{N}+\frac{1}{5} \mathrm{~J}_{10 \times 10}\right) & \frac{1}{3}\left(\mathbf{N}+\frac{1}{5} \mathrm{~J}_{10 \times 10}\right) t \\ \mathbf{0}_{10} & \frac{1}{3}\left(\mathbf{N}+\frac{1}{5} \mathbf{J}_{10 \times 10}\right)\end{array}\right]$,

where

$$
\mathbf{N}=\left[\begin{array}{cc}
\operatorname{circ}[1,-1,0,0,-1] & -\mathbf{I}_{5} \\
-\mathbf{I}_{5} & \operatorname{circ}[1,0,-1,-1,0]
\end{array}\right],
$$

being circ [.] a circulant matrix as defined in Prop. 3.

Proposition 6 (Complete graph $K_{n}$ ): For the complete graph $K_{n}$ with $n>2$ nodes, we have that:

- For $s \in(-(n-1), 1)$, system (5) is asymptotically stable.

- For $s<-(n-1)$ or $s>1$, system (5) is unstable.

- For $s=-(n-1)$, the components of the state subvector $\nu$ of system (5) asymptotically converge, in general, to $n$ different values.

Proof: Since the eigenvalues of $-\mathbf{L}_{p}(s)$ are in this case, $(n-1) s-(n-1)$ with multiplicity 1 , and $-s-(n-1)$ with multiplicity $n-1$, from Lemma 2 we have that the eigenvalues of $-\boldsymbol{\Gamma}(s)$ are $[\gamma((n-1) s-(n-1)) \pm$ $\left.\sqrt{\gamma^{2}((n-1) s-(n-1))^{2}+4((n-1) s-(n-1))}\right] / 2$ with multiplicity 1, and $[\gamma(-s-(n-1)) \pm$ $\left.\sqrt{\gamma^{2}(-s-(n-1))^{2}+4(-s-(n-1))}\right] / 2$ with multiplicity $n-1$, from which the first two items of the statement are immediately proved. For the third item, it is sufficient to observe that for large $t$,

$\exp (-\boldsymbol{\Gamma}(-(n-1)) t) \rightarrow\left[\begin{array}{cc}\mathbf{I}_{n}-\frac{1}{n} \mathrm{~J}_{n \times n} & \left(\mathbf{I}_{n}-\frac{1}{n} \mathrm{~J}_{n \times n}\right) t \\ \mathbf{0}_{n} & \mathbf{I}_{n}-\frac{1}{n} \mathrm{~J}_{n \times n}\end{array}\right]$ 
Proposition 7 (Complete bipartite graph $K_{m, n}$ ): For the complete bipartite graph $K_{m, n}=\left(V_{1} \cup V_{2}, E\right)$, where $\left|V_{1}\right|=m,\left|V_{2}\right|=n$, with $m \geq 1, n \geq 2$ (we number the nodes in $V_{1}$ from 1 to $m$ and the nodes in $V_{2}$ from $m+1$ to $m+n)$, we have that:

- For $|s|<1$, system (5) is asymptotically stable.

- For $|s|>1$, system (5) is unstable.

- For $s=-1$ and for large $t$ we have that:

$$
\begin{aligned}
& \mathbf{x}(t) \rightarrow \frac{1}{m+n}\left[\begin{array}{cc}
\mathbb{J}_{m \times m} & -\mathbb{J}_{m \times n} \\
-\mathbb{J}_{n \times m} & \mathbb{J}_{n \times n}
\end{array}\right]\left(\mathbf{x}_{0}+\boldsymbol{\nu}_{0} t\right), \\
& \boldsymbol{\nu}(t) \rightarrow \frac{1}{m+n}\left[\begin{array}{cc}
\mathbb{J}_{m \times m} & -\mathbb{J}_{m \times n} \\
-\mathbb{J}_{n \times m} & \mathbb{J}_{n \times n}
\end{array}\right] \boldsymbol{\nu}_{0} .
\end{aligned}
$$

Proof: Since the $m+n$ eigenvalues of $-\mathbf{L}_{p}(s)$ are $-n$ with multiplicity $m-1,-m$ with multiplicity $n-1$, and $-\frac{1}{2}(n+m) \pm \frac{1}{2} \sqrt{(n-m)^{2}+4 m n s^{2}}$ with multiplicity 1 , from Lemma 2 we conclude that the $2(m+n)$ eigenvalues of $-\boldsymbol{\Gamma}(s)$ are $\left[-\gamma n \pm \sqrt{(\gamma n)^{2}-4 n}\right] / 2$ and $\left[-\gamma m \pm \sqrt{(\gamma m)^{2}-4 m}\right] / 2$ with multiplicity $m-1$ and $n-1$, respectively, and

$$
\begin{aligned}
\gamma & {\left[-n-m \pm \sqrt{(n-m)^{2}+4 m n s^{2}}\right] / 4 } \\
\pm & \frac{1}{2} \frac{\sqrt{\gamma^{2} m n s^{2}+\left(\mp \frac{\gamma^{2}}{2}(n+m) \pm 2\right) \sqrt{(n-m)^{2}+4 m n s^{2}}}}{+\frac{\gamma^{2}}{2}\left(n^{2}+m^{2}\right)-2(n+m)},
\end{aligned}
$$

with multiplicity 1 . By noticing that all the eigenvalues are negative only for $|s|<1$, we obtain the first two items of the statement. The third item follows from Prop. 1.

Proposition 8 (Hamming graph $H(d, q)$ ): For the Hamming graph $H(d, q)$, with $n=q^{d}$ nodes and $d \geq 1, q \geq 2$, we have that:

- For $s \in(-(q-1), 1)$, system (5) is asymptotically stable.

- For $s<-(q-1)$ or $s>1$, system (5) is unstable.

- For $s=-(q-1)$ :

- If $q=2$, for large $t$ the state vector of system (5) obeys (6).

- If $q>2$, the components of the state subvector $\nu$ of system (5) asymptotically converge, in general, to $n$ different values.

Proof: Since the $n$ eigenvalues of the adjacency matrix of the Hamming graph are $(q-1) d-q k$ with multiplicity $\Pi(k)=\left(\begin{array}{l}d \\ k\end{array}\right)(q-1)^{k}, k \in\{0,1, \ldots, d\}$ [16, Sect. 12.4.1] and the graph is $d(q-1)$-regular, the eigenvalues of $-\mathbf{L}_{p}(s)$ are $-d(q-1)+((q-1) d-q k) s$ with multiplicity $\Pi(k)$. Then, from Lemma 2, the eigenvalues of $-\boldsymbol{\Gamma}(s)$ are $[\gamma(-d(q-1)+$ $\underline{((q-1) d-q k) s) \pm \sqrt{\gamma^{2}(-d(q-1)+((q-1) d-q k) s)^{2}}}$ $\overline{+4(-d(q-1)+((q-1) d-q k) s)}] / 2$, again with multiplicity $\Pi(k)$. By noticing that all these eigenvalues are negative only for $s \in(-(q-1), 1)$, we obtain the first two items of the statement. For the first bullet of the third item, note that $H(d, 2)=Q_{d}$, and for the second bullet that $H(1, q)=K_{q}$ with $q>2$ (cf. [21, Sect. 9.2]).

For the reader's convenience, all the main results found in this section are summarized in Table I.

\begin{tabular}{|l|c|}
\hline Graph name & Asympt. stab. for : \\
\hline \hline Path graph $P_{n}, n \geq 2$ & $|s|<1$ \\
\hline Cycle graph $C_{n}, n>2, n$ even & $|s|<1$ \\
\hline Cycle graph $C_{n}, n>2, n$ odd & $s \in(\mu, 1)$ \\
\hline$m$-cube, $Q_{m}, n=2^{m}>4$ & $|s|<1$ \\
\hline Petersen graph $J(5,2,0)$ & $s \in(-3 / 2,1)$ \\
\hline Complete graph $K_{n}, n>2$ & $s \in(-(n-1), 1)$ \\
\hline Complete bipartite graph $K_{m, n}, m \geq 1, n \geq 2$ & $|s|<1$ \\
\hline Hamming graph $H(d, q), d \geq 1, q \geq 2$ & $s \in(-(q-1), 1)$ \\
\hline
\end{tabular}

TABLE I

SUMMARY OF THE STABILITY PROPERTIES OF SYSTEM (5) FOR SEVEN SPECIAL FAMILIES OF GRAPHS. CONSENSUS IS REACHED ASYMPTOTICALLY IN ALL CASES FOR $s=1$.

Remark 1 (The second-order Laplacian pencil): In order to study the stability properties of system (5) for general graphs $\mathcal{G}$, it is convenient to interpret $\boldsymbol{\Gamma}(s)$ as a matrix pencil in the indeterminate $s$ : note, in fact, that we can rewrite $\boldsymbol{\Gamma}(s)$ as,

$$
\boldsymbol{\Gamma}(s)=\left[\begin{array}{cc}
\mathbf{0}_{n} & -\mathbf{I}_{n} \\
\mathbf{D} & \gamma \mathbf{D}
\end{array}\right]-s\left[\begin{array}{cc}
\mathbf{0}_{n} & \mathbf{0}_{n} \\
\mathbf{A} & \gamma \mathbf{A}
\end{array}\right],
$$

which we call the second-order Laplacian pencil to distinguish it from the Laplacian pencil $\mathbf{L}_{p}(s)$ studied in [10]. It is easy to verify that:

- The second-order Laplacian pencil is a regular pencil (in fact, we always have $\operatorname{det}(\boldsymbol{\Gamma}(s))=\operatorname{det}(\mathbf{D}) \neq 0$ for $s=0$, cf. [22, Def. 4.7]).

- Let $\operatorname{spec}[\cdot]$ denote the spectrum of a matrix pencil and $\operatorname{spec}_{\mathrm{F}}[\cdot]$ the spectrum of a matrix pencil without the eigenvalues at infinity. Then, according to [22, Prop. 4.6], we have that:

$$
\operatorname{spec}[\boldsymbol{\Gamma}(s)]=\operatorname{spec}_{\mathrm{F}}\left[-\mathbf{L}_{p}(s)\right] \cup(\infty)^{r} \cup(-\infty)^{n},
$$

where $r=n-\operatorname{deg}\left[\operatorname{det}\left(\mathbf{L}_{p}(s)\right)\right]$ and $(\cdot)^{r}$ indicates an eigenvalue with algebraic multiplicity $r$. Since the Laplacian pencil has all real eigenvalues then from (8) also the second-order Laplacian pencil has all real eigenvalues.

It has been shown in [10], that if the graph $\mathcal{G}$ is regular, the smallest and second smallest eigenvalue in modulus of the negated Laplacian pencil, are the values of $s$ for which the system $\dot{\mathbf{x}}=-\mathbf{L}_{p}(s) \mathbf{x}$ is marginally stable, and that it is asymptotically stable for all $s$ between these two values. We have not found so far counterexamples to this property for system (5): however, a formal proof of this statement is the subject of ongoing research.

\section{WORKED EXAMPLES}

The theory presented in the previous section is here illustrated with the help of two numerical examples. 

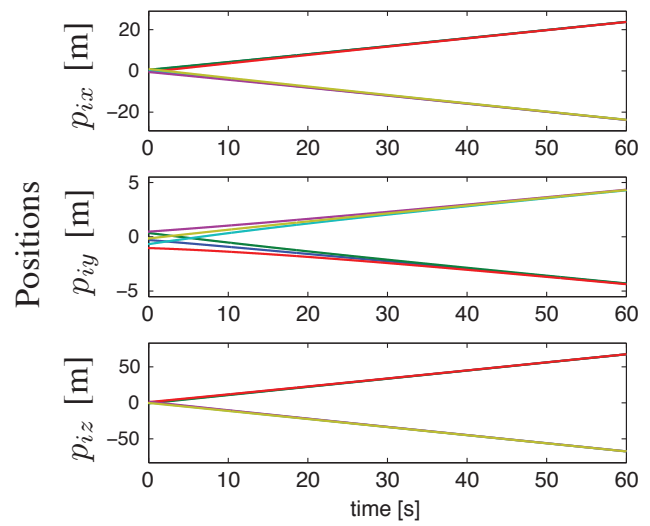

(a)

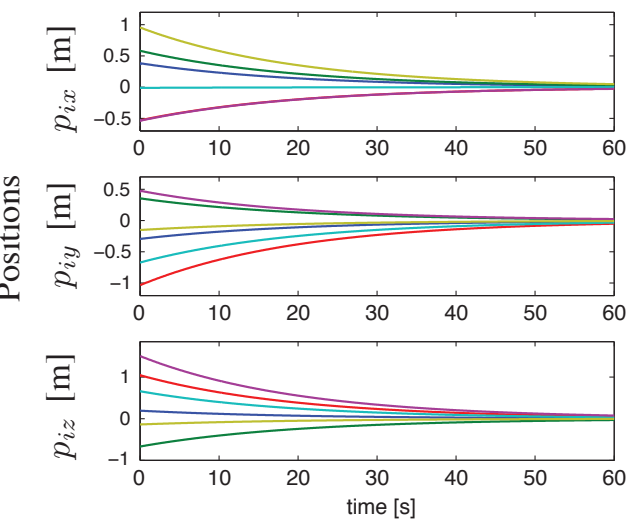

(c)

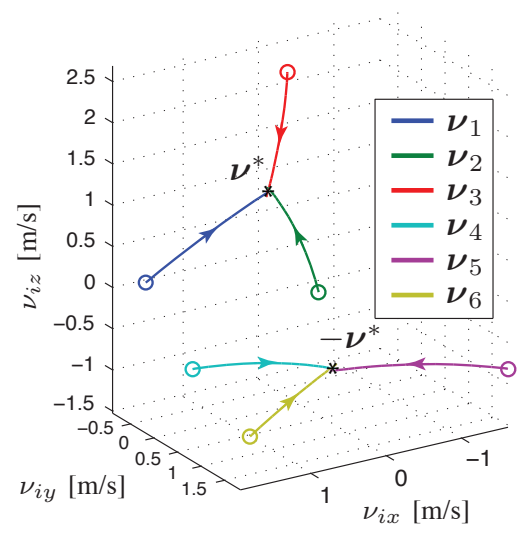

(b)

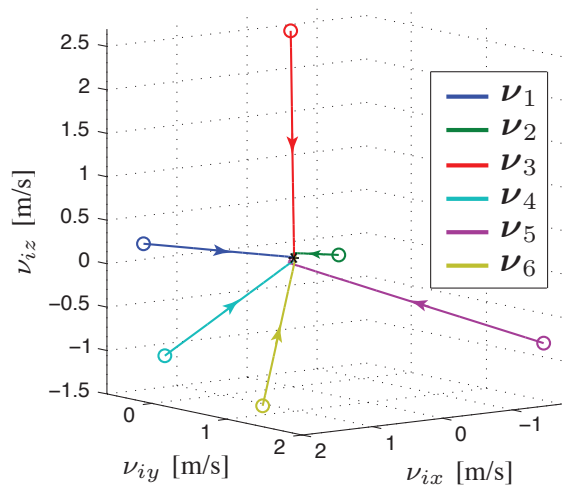

(d)

Fig. 1. Example 1 - coordination of a team of UAVs: (a), (c) time evolution of the positions, and (b), (d) evolution of the velocities of the 6 UAVs interacting via the communication graph $K_{3,3}$ : in (a)-(b), we set $s=-1$, in (c)-(d) we set $s=0$, at all times. In (b), (d), the initial velocities of the vehicles are marked with circles: note that in (a), (c) the same color convention as in (b), (d) has been adopted.

\section{A. Example 1: coordination of a team of quadrotor UAVs}

Consider a team of $n$ quadrotor UAVs modeled as $\ddot{\mathbf{p}}_{i}=\mathbf{u}_{i}, i \in\{1, \ldots, n\}$, where $\mathbf{p}_{i}=\left[p_{i x}, p_{i y}, p_{i z}\right]^{T} \in \mathbb{R}^{3}$ denotes the 3-D position of the $i$-th UAV in a fixed global reference frame and $\mathbf{u}_{i} \in \mathbb{R}^{3}$ its acceleration input. The collective dynamics of the $n$ UAVs adopting control (4), can be written in compact form as

$$
\dot{\mathbf{q}}=\left(-\boldsymbol{\Gamma}(s) \otimes \mathbf{I}_{3}\right) \mathbf{q},
$$

where $\mathbf{q}=\left[\mathbf{p}_{1}^{T}, \boldsymbol{\nu}_{1}^{T}, \ldots, \mathbf{p}_{n}^{T}, \boldsymbol{\nu}_{n}^{T}\right]^{T} \in \mathbb{R}^{6 n}$ and $\boldsymbol{\nu}_{i}=$ $\left[\nu_{i x}, \nu_{i y}, \nu_{i z}\right]^{T} \in \mathbb{R}^{3}$ is the velocity vector of the $i$-th UAV.

Figs. 1(a)-(b) show the time history of the positions and the velocity profiles of $n=6$ UAVs communicating over the graph $K_{3,3}$ : in the simulation, the damping parameter $\gamma=20, s=-1$ at all times, and the initial condition $\mathbf{q}_{0}$ has been chosen at random. Figs. 1(c)-(d) report again the timehistory of the positions and the velocity profiles of the UAVs, but now $s=0$ at all times $\left(\mathbf{q}_{0}\right.$ and all the other simulation parameters have been left unchanged). As it is evident from Fig. 1, in the case of $s=-1$ the velocities of UAVs 1,2 and 3 converge to $\boldsymbol{\nu}^{*}=[0.396,-0.0702,1.128]^{T}$ and the velocities of UAVs 4,5 , and 6 converge to $-\boldsymbol{\nu}^{*}$ (cf. Prop. 1): for $s=0$, instead, all the velocities converge to zero being system (9) asymptotically stable (cf. Prop. 7).

\section{B. Example 2: network of temperature sensors}

Consider a static network of $n$ sensors in a 2-D bounded environment $\mathscr{Q}$, locally measuring temperatures $x_{i}$ (in $\mathrm{C}^{\circ}$ ) and gradients of temperatures $\nu_{i}, i \in\{1, \ldots, n\}$ (see the illustration in Fig. 2). Sensor $i$ collects noisy measurements with variance $\sigma_{i}^{2}$ and interacts with the other sensors through the visibility graph $\mathcal{G}_{\text {vis }}$ : in this connected undirected graph, two sensors are neighbors if they are visible to each other, i.e. $\{i, j\} \in E$ if the closed segment from $i$ to $j$ is contained in $\mathscr{Q}$. The set of neighbors of agent $i$ in $\mathcal{G}_{\text {vis }}$ is denoted by $\mathcal{N}_{\text {vis }}(i)$. By taking the variance of the measurements into account, the state vector $\left[x_{i}, \nu_{i}\right]^{T}$ of sensor $i$ can be updated according to the following inverse-variance

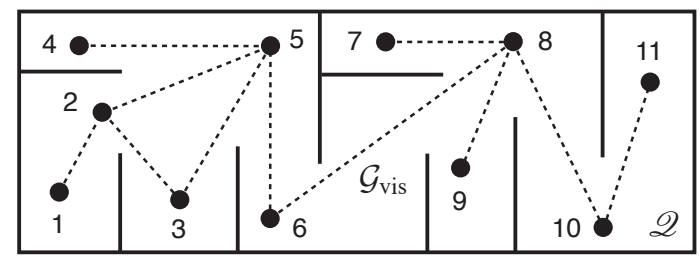

Fig. 2. Example 2 - cooperative temperature measurement: the sensors are marked with black disks and dashed lines indicate the communication pattern in the bounded environment $\mathscr{Q}$ (e.g., the plan of a museum). 


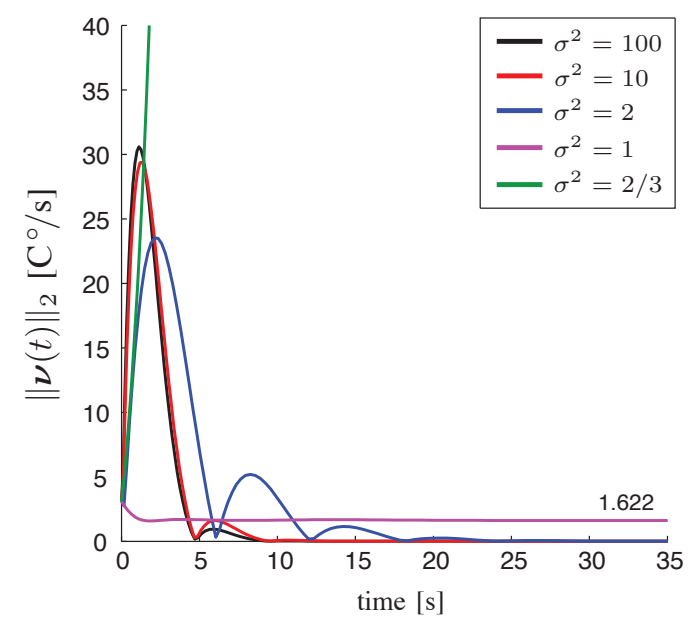

Fig. 3. Example 2: time evolution of the Euclidean norm of the state subvector $\boldsymbol{\nu}(t)$ of system (11), for different values of variance $\sigma^{2}$.

weighted sum-like criterion:

$$
\begin{aligned}
\dot{x}_{i} & =\nu_{i}, \quad i \in\{1, \ldots, n\}, \\
\dot{\nu}_{i} & =\left[\sum_{j \in \mathcal{N}_{\text {vis }}(i)}\left(\frac{1}{\sigma_{j}^{2}}+\frac{1}{\sigma_{i}^{2}}\right)\right]^{-1} \sum_{j \in \mathcal{N}_{\text {vis }}(i)}\left[\left(\frac{1}{\sigma_{j}^{2}} x_{j}-\frac{1}{\sigma_{i}^{2}} x_{i}\right)\right. \\
& \left.+\gamma\left(\frac{1}{\sigma_{j}^{2}} \nu_{j}-\frac{1}{\sigma_{i}^{2}} \nu_{i}\right)\right] .
\end{aligned}
$$

If we now assume for simplicity that $\sigma_{i}=1, \sigma_{j}=\sigma, \forall j \neq i$, and define $s \triangleq 1 / \sigma^{2}$, then (10) reduces to:

$$
\begin{aligned}
& \dot{x}_{i}=\nu_{i}, \quad i \in\{1, \ldots, n\} \\
& \dot{\nu}_{i}=\frac{1}{\left|\mathcal{N}_{\text {vis }}(i)\right|(s+1)} \sum_{j \in \mathcal{N}_{\text {vis }}(i)}\left[\left(s x_{j}-x_{i}\right)+\gamma\left(s \nu_{j}-\nu_{i}\right)\right] .
\end{aligned}
$$

By collecting the dynamics of all sensors together, we end up with the following system:

$$
\left[\begin{array}{c}
\dot{\mathbf{x}} \\
\dot{\boldsymbol{\nu}}
\end{array}\right]=-\left[\begin{array}{cc}
\mathbf{0}_{n} & -\mathbf{I}_{n} \\
\frac{1}{s+1} \mathbf{L}_{\mathrm{rw}}(s) & \frac{\gamma}{s+1} \mathbf{L}_{\mathrm{rw}}(s)
\end{array}\right]\left[\begin{array}{c}
\mathbf{x} \\
\boldsymbol{\nu}
\end{array}\right],
$$

where $\mathbf{x}=\left[x_{1}, \ldots, x_{n}\right]^{T}, \boldsymbol{\nu}=\left[\nu_{1}, \ldots, \nu_{n}\right]^{T}$ and $\mathbf{L}_{\mathrm{rw}}(s)=$ $\mathbf{I}_{n}-\mathbf{D}^{-1} \mathbf{A} s$. For $s=1, \mathbf{L}_{\mathrm{rw}}(s)$ is referred to as the normalized Laplacian in [23], and as the subscript suggests it is closely related to a random walk (in fact, the transition probability matrix of a random walk on $\mathcal{G}_{\text {vis }}$ is $\mathbf{D}^{-1} \mathbf{A}$ ). Note that unless $\mathcal{G}_{\text {vis }}$ is regular, matrix $\mathbf{L}_{\mathrm{rw}}(s)$ is non-symmetric.

Fig. 3 shows the time evolution of the Euclidean norm of $\boldsymbol{\nu}(t)$ for five different values of variance $\sigma^{2}$, for the 11-node visibility graph $\mathcal{G}_{\text {vis }}$ reported in Fig. 2. The initial condition is $\mathbf{x}_{0}=21 \cdot \mathbb{1}, \boldsymbol{\nu}_{0}=$ $[1.1706,0.4759,1.4122,0.0226,-0.0479,1.7013,-0.5097$, $-0.0029,0.9199,0.1498,1.4049]^{T}$ chosen at random, and $\gamma=1.5$. Note that for $\sigma^{2}=1$ and for large $t$, $\|\boldsymbol{\nu}(t)\|_{2} \rightarrow\left\|\mathbb{1} \mathbf{w}^{T} \boldsymbol{\nu}_{0}\right\|_{2} \simeq 1.622$ where $\mathbf{w} \in \mathbb{R}^{n}$ is a nonnegative left eigenvector of $-\mathbf{L}_{\mathrm{rw}}(1)$ associated with the zero eigenvalue and $\mathbf{w}^{T} \mathbb{1}=1$. Instead, for $\sigma^{2} \in\{2,10,100\}$ system (11) is asymptotically stable, and for $\sigma^{2}=2 / 3$ the system is unstable.

\section{CONCLUSIONS AND FUTURE WORK}

In this paper we have presented an extension of the parametric consensus protocol to agents modeled as double integrators. The stability properties of the new protocol in terms of the real parameter " $s$ ", have been studied for some relevant graph topologies and the theory has been illustrated with the help of two worked examples.

In future research, as mentioned in Remark 1, we aim at establishing general stability conditions for arbitrary (weighted) undirected communication networks, and at addressing the more challenging case of directed topologies. We are also interested in studying the robustness of the proposed protocol when the states $\nu_{j}$ of the neighbors of node $i$ are not perfectly known.

\section{REFERENCES}

[1] M. Mesbahi and M. Egerstedt. Graph Theoretic Methods in Multiagent Networks. Princeton University Press, 2010.

[2] R. Olfati-Saber and R.M. Murray. Consensus problems in networks of agents with switching topology and time-delays. IEEE Trans. Automat. Contr., 49(9):1520-1533, 2004.

[3] S. Kar and J.M.F. Moura. Distributed Consensus Algorithms in Sensor Networks With Imperfect Communication: Link Failures and Channel Noise. IEEE Trans. Signal Proces., 57(1):355-369, 2009.

[4] M. Egerstedt, S. Martini, M. Cao, K. Camlibel, and A. Bicchi. Interacting with Networks: How Does Structure Relate to Controllability in Single-Leader, Consensus Networks? IEEE Contr. Syst. Mag., 32(4):66-73, 2012.

[5] S. Zhang, M. Cao, and M.K. Camlibel. Upper and lower bounds for controllable subspaces of networks of diffusively coupled agents IEEE Trans. Automat. Contr., 59(3):745-750, 2014.

[6] G. Parlangeli and G. Notarstefano. On the reachability and observability of path and cycle graphs. IEEE Trans. Automat. Contr., 57(3):743748, 2012.

[7] J. Ren, W.-X. Wang, B. Li, and Y.-C. Lai. Noise bridges dynamical correlation and topology in coupled oscillator networks. Phys. Rev. Lett., 104(5):058701.1-058701.4, 2010.

[8] S. Sundaram and C.N. Hadjicostis. Distributed function calculation and consensus using linear iterative strategies. IEEE J. Sel. Area Comm., 26(4):650-660, 2008.

[9] F. Morbidi. The deformed consensus protocol. Automatica, 49(10):3049-3055, 2013.

[10] F. Morbidi. The Laplacian Pencil and its Application to Consensus Theory. Submitted, 2013.

[11] D. Cvetković, P. Rowlinson, and S.K. Simić. Signless Laplacians of finite graphs. Linear Algebra Appl., 423:155-171, 2007.

[12] L. Zhang, H. Jiang, and S. Guo. Cluster Anticonsensus of Multi-agent Systems Based on the Q-Theory. Discrete Dyn. Nat. Soc., 2014:ID 254749, 7 pages, 2014.

[13] W. Ren and E. Atkins. Distributed multi-vehicle coordinated control via local information exchange. Int. J. Robust Nonlin. Contr., 17(1011):1002-1033, 2007.

[14] W. Yu, G. Chen, and M. Cao. Some necessary and sufficient conditions for second-order consensus in multi-agent dynamical systems Automatica, 46(6): 1089-1095, 2010.

[15] F. Goldberg and S. Kirkland. On the sign patterns of the smallest signless Laplacian eigenvector. Linear Algebra Appl., 443:66-85, 2014.

[16] A.E. Brouwer and W.H. Haemers. Spectra of graphs. Springer, 2012.

[17] S. Fallat and Y.-Z. Fan. Bipartiteness and the least eigenvalue of signless Laplacian of graphs. Linear Algebra Appl., 436(9):3254 3267,2012

[18] C. Altafini. Consensus problems on networks with antagonistic interactions. IEEE Trans. Automat. Contr., 58(4):935-946, 2013.

[19] C. Godsil and G. Royle. Algebraic graph theory. Springer, 2001.

[20] P.J. Davis. Circulant Matrices. New York, Chelsea, 2nd edition, 1994.

[21] A.E. Brouwer, A.M. Cohen, and A. Neumaier. Distance-Regular Graphs. Springer, 1989.

[22] J.W. Demmel. Applied numerical linear algebra. SIAM, 1997.

[23] U. von Luxburg. A tutorial on spectral clustering. Stat. Comput., 17(4):395-416, 2007. 